\title{
Reliability and Validity of the Korean Version of Health Anxiety Questionnaire
}

\author{
Kyu-Sic Hwang ${ }^{1,2}$, Seung-Ho Jang ${ }^{1,2}$, Hye-Jin Lee ${ }^{3}$, and Sang-Yeol Lee ${ }^{1,2,4 凶}$ \\ ${ }^{1}$ Department of Psychiatry, Wonkwang University Hospital, Iksan, Republic of Korea \\ ${ }^{2}$ Wonkwang Brain-Behavior Mental Health Institute, Iksan, Republic of Korea \\ ${ }^{3}$ Department of Public Health, Wonkwang University Graduate School, Iksan, Republic of Korea \\ ${ }^{4}$ Department of Psychiatry, School of Medicine, Wonkwang University, Iksan, Republic of Korea
}

Objective Health anxiety can be defined by concern about health in the absence of a pathology. The Health Anxiety Questionnaire (HAQ) based on the cognitive-behavioral model can be useful for evaluating the severity and the structure of health anxiety. This study aims to verify the reliability and validity of Korean version of HAQ (K-HAQ).

Methods For reliability, test-retest reliability and internal consistency were analyzed. For construct validity, exploratory factor analysis (EFA) and confirmatory factor analysis (CFA) were conducted. Receiver Operating Characteristic (ROC) analysis was performed to identify the optimal cut-off score.

Results Cronbach's alpha was 0.92 , and $\mathrm{r}$ value of test-retest reliability was 0.84 . In the EFA, 4- and 5-factor model showed cumulative percentile of variance of $60 \%$ or more. In the CFA, the 4 -factor model was found to be the most appropriate and simplest $\left(\chi^{2}=397.33\right.$, $\mathrm{df}=187, \mathrm{CFI}=0.909, \mathrm{TLI}=0.888, \mathrm{RMSEA}=0.077$ ). In the ROC analysis, the cut-off score was 20 points.

Conclusion It is expected that K-HAQ can be helpful to evaluate the severity of health anxiety and make therapeutic plans because KHAQ can help explore the cognitive, emotional, and behavioral structure of health anxiety by each factor.

Psychiatry Investig 2018;15(10):976-983

Key Words Health anxiety, Hypochondriasis, Somatic symptom disorder, Illness anxiety disorder.

\section{INTRODUCTION}

What is health anxiety? Health anxiety can be defined by concern about health in the absence of a pathology or excessive worries when there is some degree of pathology. In the light of psychiatric diagnosis, health anxiety can belong to the hypochondriasis based on the Diagnostic and Statistical Manual of Mental Disorders, 4th Edition, Text Revision (DSM-IVTR), or the somatic symptom disorder or the illness anxiety disorder based on the Diagnostic and Statistical Manual of Mental Disorders, Fifth Edition (DSM-5). ${ }^{1,2}$ In general, at first people with health anxiety often visit the departments of inter-

Received: May 14, 2018 Revised: July 10, 2018

Accepted: July 25, 2018

$\triangle$ Correspondence: Sang-Yeol Lee, MD, PhD

Department of Psychiatry, School of Medicine, Wonkwang University, 895

Muwang-ro, Iksan 54538, Republic of Korea

Tel: +82-63-859-1044, Fax: +82-63-857-1043

E-mail: psysangyeol@hanmail.net

(a) This is an Open Access article distributed under the terms of the Creative Commons Attribution Non-Commercial License (https://creativecommons.org/licenses/bync/4.0) which permits unrestricted non-commercial use, distribution, and reproduction in any medium, provided the original work is properly cited. nal medicine rather than the department of psychiatry because the patients believe that they have real disease or existing symptoms and the internal medicine or other surgery departments can solve their problems. ${ }^{3}$ However, their anxiety or related symptoms rarely disappear regardless of the presence of real disease or symptoms. They have even visited other hospitals, and their anxiety becomes higher and higher. The things these patients need would be the antianxiety drugs or psychotherapy to be able to reduce the level of their anxiety, but the patients and the experts don't know the presence of health anxiety and even the patients don't admit health anxiety. Therefore, it is important for the patients to understand how much and what kind of health anxiety they have.

However, how can we know and measure health anxiety? In general, checking one's health anxiety can be known through self-report scale related hypochondriasis. Previously developed well-known health anxiety scales include Whiteley Index (WI) by Pilowsky and Illness Attitude Scale (IAS) by Kellner and his colleagues. ${ }^{4-6}$ The 14 -item WI assesses health anxiety through dimensional approach. The items in the WI 
are based on clinician's experiences of severe health anxiety, and the WI is comprised of three factors-bodily preoccupation, disease phobia, and disease conviction. But, the subsequent studies showed inconsistent results regarding factor structure of the WI. ${ }^{7}$ The IAS is a 29-item scale that has nine subscales, but the empirical data have not consistently supported the factor structure by the originators. ${ }^{8}$ Moreover, both scales above were neither developed based on a theoretical model nor validated for the patients with hypochondriasis.

Why does the health anxiety scale need the theoretical model? Barsky, Geringer, and Wool consider hypochondriasis as a cognitive and perceptual disability. ${ }^{9}$ In other words, people with hypochondriasis tend to perceive and interpret normal body sensations, symptoms of minor illnesses, or emotional physical reactions as very harmful, and consequently become obsessed with the symptoms. From this perspective, the patients with hypochondriasis need to recognize the cognitive process of their health anxiety. In general, there are recent previous studies that cognitive-behavioral therapy is effective when treating anxiety-related disorders. ${ }^{10,11}$ Specifically, in treating health anxiety, previous studies found that the treatments based on the cognitive-behavioral model appeared promising in treating health anxiety. ${ }^{12-14}$ Therefore, if we can identify the cognitive structure of health anxiety through the scale, it will help to plan the future treatment plan.

The Health Anxiety Questionnaire (HAQ) can be the scale that help to identify cognitive and behavioral structure of the health anxiety and to make the therapeutic plans. Lucock and Morley ${ }^{15}$ in 1996 developed the HAQ that was based on the cognitive-behavioral model. The model of anxiety can be divided into the development of health anxiety and the maintenance of persistent health anxiety. ${ }^{16}$ Although most of studies about hypochondriasis have focused on quantifying the somatic symptoms and the level of anxiety, it appeared not to be helpful to predict the future progress and make a therapeutic plans. However, the health anxiety can result from dysfunctional belief that negatively interpret the symptom-related important events. At the heart of these cognitive errors is the confirmatory bias that can act in perception, which can lead to selective attention and interpretation, and consequently strengthen individual anxiety. ${ }^{17}$ There is evidence to support that people with anxiety problems have cognitive bias. ${ }^{7}$ People with anxiety problems, especially those related to health, are more vulnerable to clues related to health threats. Thus they become more preoccupied with health-related symptoms and lead to reassurance-seeking behavior. ${ }^{17}$ The reassurance behaviors can cause a short-term reduction of anxiety, but the behaviors are negatively reinforced. Additionally, they might find other medical consultations, ask friends or family mem- bers, or look for medical books. The dysfunctional beliefs, cognitive bias, and reassurance behaviors discussed above occur in the cognitive processes of individuals. If this cognitive process can be modified, it is expected that it will reduce the level of health anxiety and change the behavior accordingly. In developing the HAQ, some of items from the IAS were included in modified form because the items can describe symptom of health anxiety in a cognitive way. Thus, the HAQ constructed 21 items that can be grouped 4 -factor based on the cognitive behavioral model. In the original HAQ study, its reliability, validity, and specificity appeared to be excellent.

In South Korea, the Korean version of HAQ (K-HAQ) has not been validated yet. In the light of cognitive-behavioral model, we authors expect that the K-HAQ will be useful to check the level of health anxiety and explore the cognitive structure of health anxiety. ${ }^{15}$ As with the original HAQ study, we authors hypothesize that K-HAQ will have 4-factor structure because the original HAQ was developed based on the theoretical model and have shown excellent validity in the both normal and patients with hypochondriasis group. ${ }^{15}$ Methodologically, we will testify reliability and validity of the K-HAQ. For the reliability, the test-retest reliability and internal consistency will be performed by correlation analysis and Cronbach's alpha calculation. For the validity, the concurrent validity and construct validity will be performed by correlation analysis and both exploratory and confirmatory fator analysis. Additionally, we will investigate the optimal cut-off score through Receiver Operating Curve (ROC) analysis. Finally, the purpose of this study is to validate and utilize the KHAQ in clinical setting.

\section{METHODS}

\section{Development of the Korean version of the Health Anxiety Questionnaire (K-HAQ)}

The HAQ consists of total 21 items describing health anxiety related symptoms, and it uses a four-point Likert scale. The four points 'not at all or rarely', 'sometimes', 'often', and 'most of the time' were scored from o to 3, respectively. Before starting the validation study for K-HAQ, the authors received the approval for the Korean validation study from the original authors. First, the authors translated the original English version into Korean, and then did back-translation by a bilingual (English and Korean) expert. Next, the authors and three other psychiatrists compared the two versions (original and back-translated one). After considering Korean cultural adaptation, the final version was determined by the authors.

\section{Participants}

As a normal group, initially total 218 subjects who consist 
of college students or public servants registered. The inclusion and exclusion criteria for the normal group are as follows. Participants were from 18 to 65 years of age. Through the initial general survey (gender, age, residence, marital status, education level, medical and psychiatric history), those who had psychiatric history or underlying severe and apparent medical history (i.e., brain disease, cancer, heart disease, etc.) were excluded from the data analysis. In addition, participants were those with a Somatic Complaints-Health Concern (SOM-H) Scale score of less than 65 points in the Personality Assessment Inventory (PAI). Finally, data from 189 participants were used to analyze results. The 38 participants of them received the same tests again to investigate test-retest reliability two weeks after the initial test. As a patient group (named as "hypochondriasis group" in this study), the participants are total 43 subjects. The patients are psychiatric outpatients diagnosed Hypochondriasis, Somatic Symptoms Disorder, or Illness Anxiety Disorder based on DSM-IV-TR and DSM-5 by psychiatrists. ${ }^{1,2}$ This study was approved by the Institutional Review Board of Wonkwang University Hospital (No. WKUH 2017-04-007-001), and all the participants were given written informed consent before starting the study.

\section{Measures}

To evaluate concurrent validity, two other scales were included below.

\section{Illness Attitudes Scale}

The IAS was originally developed by Kellner et al. ${ }^{5}$ in $1987 .{ }^{6}$ The IAS measures fears, attitudes, and beliefs with health concerns and abnormal illness behavior. It is four-point Liker scale and consists of total 29 items that can be divided by 9 sub-scales. We used the Korean version of IAS (K-IAS), which previously have shown high internal consistency (Cronbach's alpha $=0.86$ ) and concurrent validity compared with the Whiteley Index ( $\mathrm{r}=0.653) .{ }^{18}$ In this study, the Cronbach's alpha was 0.870 , which means "Good" internal consistency, and concurrent validity compared with the Somatic Complaints-Health concern (SOM-H) of Personality Assessment Inventory (PAI) also showed "Medium" effect size $(r=0.356) .{ }^{19,20}$

\section{Somatic Complaints-Health Concern Scale of Personality Assessment of Inventory \\ The PAI can assess both personality and psychopathology, and it includes 11 clinical scales to explain psychopathological symptoms such as depression, anxiety, schizophrenia, and so on. ${ }^{19}$ One of clinical scales is SOM scale that can be divided by three subscales-Conversion, Somatization, and Health Con- cern. Specifically, SOM-Health Concern (SOM-H) scale can measure the degree of a preoccupation with health and phys-}

ical function. In this study, we used SOM-H items of the Korean version of PAI (K-PAI). The reliability of SOM-H scale in K-PAI showed the value of $\alpha$ with 0.60 (here, $\alpha$ refers to the estimation of mean value for the split-half reliability). ${ }^{21}$ The concurrent validity of SOM compared with Hypochondriasis scale of Minnesota Multiphasic Personality Inventory (MMPI) showed "Large" effect size $(\mathrm{r}=0.53) .{ }^{21}$ In this study, the Cronbach's alpha was 0.790 , which means "Acceptable" internal consistency, and concurrent validity compared with the K-IAS also showed "Medium" effect size $(\mathrm{r}=0.356){ }^{20}$

\section{Statistical analysis}

First, both descriptive statistics and frequency analysis were used to analyze all the demographic data. To verify the reliability of the K-HAQ, Cronbach's alpha as the internal consistency was calculated. It can often be described as "Acceptable" when Cronbach's alpha is between 0.70 and 0.80 , "Good" when it is between 0.80 and 0.90 , and "Excellent" when it is above 0.90 . As the reliability of test-retest, the Pearson's correlation analysis was performed. According to Cohen, the effect size is "Low" if the value of $r$ varies around 0.1, "Medium" if $r$ varies around 0.3 , and "Large" if $r$ varies more than $0.5 .^{20}$ Next, to verify the validity of the K-HAQ, the Pearson's correlation analysis was performed as the concurrent validity. As the construct validity, the Exploratory Factor Analysis (EFA) was performed to explore the underlying structure of the entire items in the K-HAQ and find out a relatively large set of variables as a latent variable. In the EFA, we adopted Maximum Likelihood (ML) and Oblique rotation (Direct Oblimin). Then, Confirmatory Factor Analysis (CFA) was conducted to evaluate the goodness-of-fit for the models that were previously explored in the EFA. The goodness-of-fit indexes used in this study were Tucker-Lewis Index (TLI), Comparative Fit Index (CFI), and Root Mean Square Error of Approximation (RMSEA). When TLI or CFI is 0.90 or more, the goodness-offit for the model can be considered as "Acceptable".22,23 When the RMSEA is 0.08 or less, the goodness-of-fit for the model can be considered as "Acceptable". ${ }^{24}$ Lastly, to calculate the optimal cut-off score that can discriminate between normal and hypochondriasis group, sensitivity, specificity, and predictive values were calculated by using Receiver Operating Characteristic (ROC) based on the data of hypochondriasis group. All statistical analysis was conducted by Predictive Analytics Software Statistics for Windows, version 18.0.0 (SPSS Inc., Chicago, IL, USA).

\section{RESULTS}

\section{Demographic data and descriptive statistic}

Table 1 shows the demographic characteristics and descrip- 
tive statistics of the sample by group. For the normal group, the mean age was 30.15 years with standard deviation 12.51 years. The rate of male and female was $24.90 \%$ and $75.10 \%$, respectively. The average of educated years was 14.58 years with standard deviation 1.04 year. The total mean and standard deviation score of K-HAQ were 12.76 and 9.29 points, respectively. The total mean and standard deviation score of K-IAS were 63.72 and 14.48 points, respectively. The total mean and standard deviation score of SOM-H of K-PAI were 4.70 and 2.33 points, respectively. For the hypochondriasis group, the mean age was 55.76 years with SD 16.08 years. The rate of male and female was $34.88 \%$ and $65.12 \%$, respectively. The average of educated years was 11.33 years with SD 3.48 years. The total mean and standard deviation score of K-HAQ were 32.60 and 12.08 points, respectively. The total mean and standard deviation score of K-IAS were 82.04 and 17.22 points, respectively. The total mean and standard deviation score of SOM-H were 15.35 and 4.79 points, respectively.

\section{Reliability}

The Cronbach's alpha was 0.92 as an internal consistency, which means "Excellent" internal consistency. For the test-retest reliability, the 38 participants completed the K-HAQ on two occasions with a 2-week interval. The Pearson's correlation analysis showed "Large" effect size $(r=0.840, n=38, p<0.001)$.

\section{Construct validity}

First, the value of Kaiser-Meyer-Olkin (KMO) and Bartlett's test of sphericity showed that it was possible enough to conduct factor analysis (The value of $\mathrm{KMO}=0.94 ; \chi^{2}(\mathrm{df}=210$, $\mathrm{n}=189)=2435.22, \mathrm{p}<0.001){ }^{25}$ Then, the EFA was conducted to explore the factors consisting of the items of K-HAQ. After considering the original article of $\mathrm{HAQ}$, minimum $60 \%$ of communality, and the result of scree test, we decided to explore 3-, 4-, and 5-factor structures. Table 2 shows the goodness-of-fit for three models analyzed by the EFA. The values of RMSEA were $0.073,0.061$, and 0.046 , respectively. As in the EFA, the CFA was also tested for the goodness-of-fit. The indexes for the goodness-of-fit include TLI, CFI, and RMSEA. Table 2 shows the goodness-of-fit for the three models analyzed by the CFA. Based on the CFA results according to the number of factors using the goodness-of-fit indexes, for the 3-factor model, TLI, CFI, and RMSEA were 0.871, 0.895, and 0.083 , respectively. For the 4-factor model, TLI, CFI, and RMSEA were $0.888,0.909$, and 0.077 , respectively. For the 5-factor model, TLI, CFI, and RMSEA were 0.891, 0.913, and 0.076 , respectively. The models with CFI or TLI values of 0.90 or higher and RMSEA values of 0.08 or lower were 3 -factor and 4-factor models. Comparing the 3 -factor model with the 4 -factor model, the difference between the $\chi^{2}$ values of the three-factor model and the four-factor model was significant $\left[\Delta \chi^{2}(3, \mathrm{n}=189)=12.47, \mathrm{p}<0.001\right]$, but the difference in the good-

Table 1. The demographic characteristics and descriptive statistic of the sample by group

\begin{tabular}{lcc}
\hline & Normal group $(\mathrm{N}=189)$ & Hypochondriasis group (N=43) \\
\hline Age, years: mean (SD) & $30.15(12.51)$ & $55.76(16.08)$ \\
Gender & & $15(34.88)$ \\
$\quad$ Male: mean (\%) & $47(24.90)$ & $28(65.12)$ \\
$\quad$ Female: mean (\%) & $142(75.10)$ & $11.33(3.48)$ \\
Education, years: mean (SD) & $14.58(1.04)$ & $32.60(12.08)$ \\
Total score of K-HAQ: mean (SD) & $12.76(9.29)$ & $82.04(17.22)$ \\
Total score of K-IAS: mean (SD) & $63.72(14.48)$ & $15.35(4.79)$ \\
Total score of SOM-H: mean (SD) & $4.70(2.33)$ &
\end{tabular}

K-HAQ: Korean version of Health Anxiety Questionnaire, K-IAS: Korean version of the Illness Attitude Scale, SOM-H: Somatic ComplaintsHealth concern of Korean version of Personality Assessment Inventory, SD: standard deviation

Table 2. The goodness-of-fit for the 3-, 4-, and 5-factor model by factor analysis solutions

\begin{tabular}{clcccccc}
\hline FAS & Model & Cumulative \% of variance explained & $\chi^{2}$ & $\mathrm{df}$ & TLI & CFI & RMSEA \\
\hline EFA & 3-factor & 60.50 & 299.067 & 150 & N/A & N/A & 0.073 \\
& 4-factor & 65.47 & 224.701 & 132 & N/A & N/A & 0.061 \\
& 5-factor & 69.99 & 161.637 & 115 & N/A & N/A & 0.046 \\
CFA & 3-factor & 60.50 & 432.56 & 189 & 0.871 & 0.895 & 0.083 \\
& 4-factor & 65.47 & 397.33 & 187 & 0.888 & 0.909 & 0.077 \\
& 5-factor & 69.99 & 384.86 & 184 & 0.891 & 0.913 & 0.076 \\
\hline
\end{tabular}

FAS: Factor Analysis Solutions, EFA: Exploratory Factor Analysis, CFA: Confirmatory Factor Analysis, TLI: Tucker-Lewis index, CFI: comparative fit index, RMSEA: root mean square error of approximation 
ness-of-fit was insignificant $(\Delta \mathrm{TLI}=-0.003, \Delta \mathrm{CFI}=-0.004$, $\triangle \mathrm{RMSEA}=0.001$ ). Considering a parsimony of the model, The 4 -factor model is considered to be the most appropriate one. ${ }^{26}$

Therefore, for the 4 -factor model (Supplementary Materials in the online-only Data Supplement), the factor 1 consists of questions $1,2,3,4,6,7,8,9,10,11$, and 18 and includes questions about health concerns and obsessions. The factor 1 was named as "Health worry and preoccupation" as same in the original. Factor 2 consists of questions 19, 20, and 21, and includes questions about how much of what you are doing is disturbed by these symptoms. The factor 2 was named as "Interference with life" as same in the original. The factor 3 consists of questions 14, 15, 16, 17 and contains questions related to fear of death and illness. The factor 3 was named as "Fear of illness and death" as same in the original. Lastly, the factor 4 consists of questions 5, 12, and 13, and includes questions related to behaviors to test and confirm symptoms. The factor 4 was named as "Reassurance-seeking behavior" as same in the original. Table 3 shows the total items and the factor loading of the K-HAQ items for the 4-factor by the EFA. The correlations among each factor showed a "Medium" to "Large" effect size of $0.30-0.65$. Table 4 shows the correlation values between the factors.

\section{Concurrent validity}

The correlation analysis was performed to examine the relationship between the K-HAQ and other well-known hypochondriasis scales. As a result, the total score of the K-HAQ showed a "Large" effect size in correlation analysis with the K-IAS scale ( $\mathrm{r}=0.798, \mathrm{n}=189, \mathrm{p}<0.001)$. Second, the total score of the K-HAQ showed a "Medium" effect size in the correlation analysis with the SOM-H scale of K-PAI $(r=0.359, n=$ $189, \mathrm{p}<0.001$ ). Additionally, the total score of the K-IAS also showed a "Medium" effect size in the correlation analysis with the SOM-H scale of K-PAI $(r=0.356, n=189, p<0.001)$. Table 5 shows the results of correlation analysis of K-HAQ with oth-

Table 3. Factor loading of the K-HAQ items for the 4-factor model by exploratory factor analysis

\begin{tabular}{|c|c|c|c|c|}
\hline Item & Factor 1 & Factor & Factor 3 & Factor 4 \\
\hline 01. Do you ever worry about your health? & 0.727 & -0.474 & -0.328 & -0.583 \\
\hline 02. Are you ever worried that you may get a serious illness in the future? & 0.769 & -0.438 & -0.427 & -0.473 \\
\hline 03. Does the thought of a serious illness ever scare you? & 0.807 & -0.479 & -0.497 & -0.448 \\
\hline $\begin{array}{l}\text { 04. When you notice an unpleasant feeling in your body, do you tend to find it difficult to think } \\
\text { of anything else? }\end{array}$ & 0.710 & -0.406 & -0.473 & -0.458 \\
\hline 06. If you have an ache or pain do you worry that it may be caused by a serious illness? & 0.787 & -0.380 & -0.523 & -0.643 \\
\hline 07. Do you ever find I difficult to keep worries about your health out of you mind? & 0.816 & -0.522 & -0.478 & -0.537 \\
\hline 08. When you notice an unpleasant feeling in you body, do you ever worry about it? & 0.769 & -0.476 & -0.513 & -0.632 \\
\hline $\begin{array}{l}\text { 09. When you wake up in the morning, do you find you very soon begin to worry about your } \\
\text { health? }\end{array}$ & 0.338 & -0.254 & -0.332 & -0.322 \\
\hline $\begin{array}{l}\text { 10. When you hear of a serious or the death of someone you know, does it ever make you more } \\
\text { concerned about your own health? }\end{array}$ & 0.728 & -0.366 & -0.567 & -0.503 \\
\hline $\begin{array}{l}\text { 11. When you read or hear about an illness on TV or radio, does it ever make you think you may } \\
\text { be suffering from that illness? }\end{array}$ & 0.717 & -0.410 & -0.605 & -0.564 \\
\hline 18. Do you ever feel afraid that you may have any other serious illness? & 0.680 & -0.501 & -0.492 & -0.508 \\
\hline 19. Have your bodily symptoms stopped you from working during the past six months or so? & 0.387 & -0.658 & -0.140 & -0.265 \\
\hline 20. Do your bodily symptoms stop you from concentrating on what you are doing? & 0.508 & -0.966 & -0.306 & -0.403 \\
\hline 21. Do your bodily symptoms stop you from enjoying yourself? & 0.538 & -0.812 & -0.369 & -0.338 \\
\hline 14. Do you ever feel afraid of news that reminds you of death (such as funerals, obituary notices)? & 0.445 & -0.221 & -0.642 & -0.405 \\
\hline 15. Do you ever feel afraid that you may die soon? & 0.570 & -0.353 & -0.891 & -0.438 \\
\hline 16. Do you ever feel afraid that you may have cancer? & 0.730 & -0.421 & -0.742 & -0.533 \\
\hline 17. Do you ever feel afraid that you might have heart disease? & 0.589 & -0.334 & -0.648 & -0.498 \\
\hline 05. Do you ever examine your body to find whether there is something wrong? & 0.471 & -0.386 & -0.298 & -0.571 \\
\hline $\begin{array}{l}\text { 12. When you experience unpleasant feeling in your body, do you tend to ask friends or family } \\
\text { about them? }\end{array}$ & 0.526 & -0.261 & -0.496 & -0.714 \\
\hline 13. Do you tend to read up about illness and disease to see if you may be suffering from one? & 0.569 & -0.364 & -0.417 & -0.859 \\
\hline
\end{tabular}

K-HAQ: Korean version of Health Anxiety Questionnaire, Factor 1: "Health worry and preoccupation", Factor 2: "Interference with life", Factor 3: "Fear of illness and health", Factor 4: "Reassurance-seeking behavior" 
er hypochondriasis scales for the concurrent validity.

\section{ROC analysis}

The ROC analysis was performed to find the cut-off score of K-HAQ, which can distinguish between normal adults and hypochondriasis patients. In the Area under the curve (AUC), which indicates the degree to which the scale accurately diagnoses the presence or absence of the disorder, a score of 0.50 or higher means a probability higher than chance, and a diagnosis based on the scale is useful when the value is $0.72-$ $0.92{ }^{27}$ The AUC for K-HAQ was 0.780 , suggesting diagnostic utility. For the total score, the sum of sensitivity and specificity was maximized when the cut-off score was 20 points, and the sensitivity was $71 \%$ and the specificity was $75 \%$ (Table 6 ). Table 7 summarizes the result of ROC analysis, and Figure 1 shows ROC curve. The diagonal segments in the Figure 1 are produced by ties.

\section{DISCUSSION}

In this study, we aimed to translate HAQ into Korean and evaluate the reliability and validity of K-HAQ, which can measure the level of anxiety for hypochondriasis and be helpful for

Table 4. Inter-factor correlations of 4-factor model in the K-HAQ

\begin{tabular}{lcccc}
\hline & Factor 1 & Factor 2 & Factor 3 & Factor 4 \\
\hline Factor 1 & 1.00 & & & \\
Factor 2 & 0.56 & 1.00 & & \\
Factor 3 & 0.60 & 0.30 & 1.00 & \\
Factor 4 & 0.65 & 0.39 & 0.47 & 1.00 \\
\hline
\end{tabular}

K-HAQ: Korean version of Health Anxiety Questionnaire, Factor 1: "Health worry and preoccupation", Factor 2: "Interference with life", Factor 3: "Fear of illness and health", Factor 4: "Reassuranceseeking behavior"

Table 5. Correlation analysis of K-HAQ with other hypochondriasis scales for the concurrent validity $(\mathrm{N}=189)$

\begin{tabular}{lclc}
\hline & K-HAQ & K-IAS & SOM-H \\
\hline K-HAQ & 1.00 & & \\
K-IAS & $0.798^{*}$ & 1.00 & \\
SOM-H & $0.359^{*}$ & $0.356^{*}$ & 1.00 \\
\hline
\end{tabular}

${ }^{*} \mathrm{p}<0.000$. K-HAQ: Korean version of Health Anxiety Questionnaire, K-IAS: Korean version of the Illness Attitude Scale, SOM-H: Somatic Complaints-Health concern of Korean version of Personality Assessment Inventory establishing a therapeutic plan in the clinical practice. The implications of the results are as follows.

First, as to the implications of the reliability test results, internal consistency reliability and test-retest reliability were all at a reliable level (Cronbach's alpha $=0.92, \mathrm{r}=0.84$ ). Comparing this with the original study, the alpha coefficient was 0.92 and $r$ value was 0.87 in the original study. These results suggest that the reliability of the K-HAQ measurement is a quite reasonable.

Second, the implication of the validity test results are as follows. In the construct validity testing, we explored 3-factor, 4-factor, and 5-factor structures in the EFA with reference to scree test, cumulative percentile of variance, and original HAQ study results. In the EFA results, the cumulative percentile of variance for both 3-, 4-, and 5-factors was more than $60 \%$ and the RMSEA was well below 0.08 . Next, the CFA results suggest that the 4-factor model and the 5-factor model are suitable models. However, since the difference of the goodness-of-fit index between the two models was not significant, the simpler 4-factor model was adopted. Comparing the CFA results with the original HAQ study results, the 4-factor model of this study was consistent with the original study results. Therefore, in this study, the authors named the factor names by naming the four factors as same in the original HAQ study. The factor 1 is "Health worry and preoccupation," the factor 2 is "Interference with life," the factor 3 is "Fear of illness and

Table 6. Sensitivity and specificity for a selection of cut-off points on the K-HAQ

\begin{tabular}{ccc}
\hline Cut-off $\geq$ & Sensitivity (\%) & Specificity (\%) \\
\hline 15 & 81 & 56 \\
16 & 77 & 60 \\
17 & 77 & 64 \\
18 & 74 & 68 \\
19 & 74 & 71 \\
$20^{*}$ & $71^{*}$ & $75^{*}$ \\
21 & 68 & 76 \\
22 & 68 & 77 \\
23 & 58 & 81 \\
24 & 55 & 83 \\
25 & 52 & 83 \\
\hline
\end{tabular}

*suggested optimal cut-off point. K-HAQ: Korean version of Health Anxiety Questionnaire

Table 7. Summary of ROC analysis

\begin{tabular}{ccccccc}
\hline & AUC & SEM & $95 \%$ CI & Cut-off score (points) & Sensitivity (\%) & Specificity (\%) \\
\hline K-HAQ & 0.780 & 0.040 & $0.702-0.858$ & 20 & 71 & 75 \\
\hline
\end{tabular}

ROC: Receiver Operating Characteristic, K-HAQ: Korean version of Health Anxiety Questionnaire, AUC: area under the curve, SEM: standard error of the mean, CI: confidence interval 


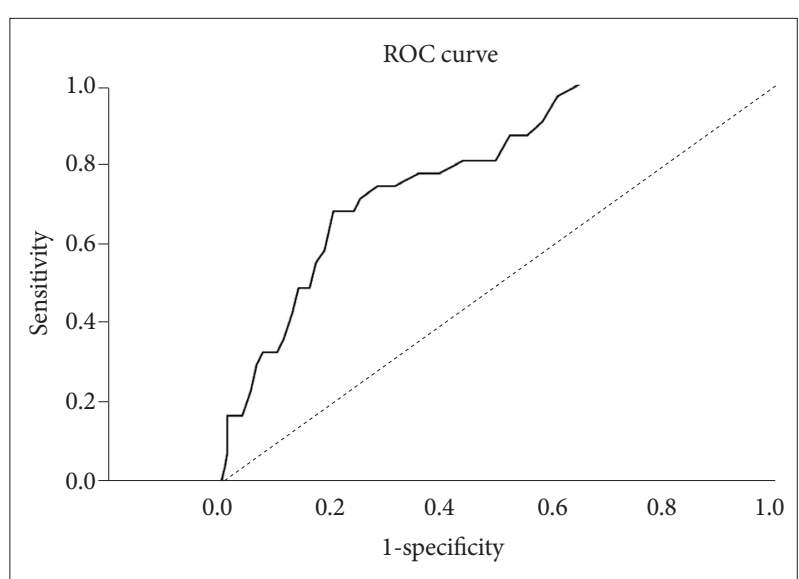

Figure 1. The ROC curve for the K-HAQ to investigate the optimal cut-off score. ROC: receiver operating characteristics, K$\mathrm{HAQ}$ : Korean version of Health Anxiety Questionnaire.

death," and the factor 4 is "Reassurance-seeking behavior." The factor 1 includes contents related to excessive worry and obsession with health, as seen in the definition of health anxiety. Especially, it includes contents that most people worry about, or worry constantly about, somatic symptoms that can be easily overtaken. The factor 2 includes questions related to the symptoms of the body, such as whether the person is unable to work, concentrate, or enjoy the pleasure. The factor 3 directly addresses the pathologies such as death, cancer, and heart disease, and contains questions about their fear. The factor 4 includes questions such as learning through a person or a book, medical examination, and so on to find out the causes of physical symptoms. The number of factors in this study and the original HAQ study were the same, but there were some differences in the items that constituted the factor 1 and factor 3. In the factor 1, this study included items $1,2,3,4,6,7$, $8,9,10,11$, and 18 , but the items 2,3 , and 10 belonging to the factor 3 of the original HAQ study were included (No. 2 items is "Are you ever worried that you may get a serious illness in the future?," No. 3 item is "Does the thought of a serious illness ever scare you?," and No. 10 item is "When you hear of a serious or the death of someone you know, does it ever make you more concerned about your own health?" The reason for this is that the factor 3 asks about "Fear of illness and death" and because questions 15, 16, and 17 more directly ask about the fear associated with illness and death. In other words, Koreans seem to think that questions 2, 3, and 10 are questions about "Health worry and preoccupation" rather than thinking that they are questions about "Fear of illness and death." The other items that consist of factor 2 and factor 4 are consistent with this study and the original HAQ study (Supplementary Materials in the online-only Data Supplement). Lastly, in the concurrent validity test, K-HAQ showed "Large" and "Medium" effect size in the correlation with K-IAS and SOM-H of K-PAI, respectively. The K-HAQ is thought to be very similar to the scales used to measure existing hypochondriasis.

Next, in the light of clinical utility, the authors investigated the cut-off score that distinguish between normal adults and patients with hypochondriasis by ROC analysis. As a result, when the total score of K-HAQ was greater than 20 points, it was classified as $71 \%$ of the patients with hypochondriasis, and $75 \%$ of the non-hypochondriasis adults could be classified. The use of cut-off score in a clinical field is expected to help diagnose hypochondriasis and identify the individual severity. In the case of the cut-off score analysis, the ROC result could not be compared with the result of the original HAQ study because the ROC analysis was not performed on the original study. However, if the authors infer the cut-off score from the mean and SD of the normal group (Table 1), the range of scores above the average of 1 to $1.5 \mathrm{SD}$ will be 22.05 to 26.70 points. In this regard, a cut-off score of 20 points could be slightly lower than the range, so further research is needed to testify the cut-off score in a clinical field.

In addition, in terms of therapeutic implications associated with cognitive-behavioral therapy, one's cognitive structure regarding health can be inferred through the factor 1 ("Health worry and preoccupation"). The factor 2 ("Interference with life") can help for the therapist in charge figure out a current level of daily life functioning of the patient. Through the factor 3 ("Fear of illness and death"), the level of negative emotional state can be inferred. Lastly, it can be understood that the style and level of coping behavior is represented by factor 4 factors ("Reassurance-seeking behavior"). It is expected helpful to make a therapeutic plan and practice cognitive behavioral therapy as K-HAQ can provide therapists with one's cognitive structure and emotional state, and the level of daily life functioning of patients with hypochondriasis depending on each factor of K-HAQ.

Finally, the limitations and future research directions of this study are as follows. Although this study has adapted and validated the original HAQ in Korean, it is required to verify the clinical utility in practical field. Especially, the present study suggested a cut-off point of K-HAQ using the data of patients with hypochondriasis, but the number of patients used in cut-off point analysis seemed to be insufficient. Therefore, future studies will need to check the health anxiety level of patients with hypochondriasis using K-HAQ and re-testify the cut-off point. Second, as K-HAQ is based on the cognitive-behavioral model, it is necessary to conduct research to apply cognitive-behavioral therapy to patients with hypochondria using $\mathrm{HAQ}$ and to verify its therapeutic efficacy. 


\section{Supplementary Materials}

The online-only Data Supplement is available with this article at https://doi.org/10.30773/pi.2018.07.25.

\section{Acknowledgments}

This study was supported by Wonkwang University in 2018.

\section{REFERENCES}

1. American Psychiatric Association. Diagnostic and Satistical Manual of Mental Disorders, Fifth ed (DSM-5). Washington DC: American Psychiatric Pub; 2013.

2. American Psychiatric Association. Diagnostic and Statistical Manual of Mental Disorders, Fourth ed Text Revision (DSM-IV-TR). Washington, DC: American Psychiatric Pub; 2000.

3. Proust M. Overview: hypochondriasis, bodily complaints, and so-matic styles. Am J Psychiatry 1983;140:273-283.

4. Pilowsky I. Dimensions of hypochondriasis. Br J Psychiatry 1967;113: 89-93.

5. Kellner R. Hypochondriasis and somatization. JAMA 1987;258:27182722.

6. Kellner R. Abridged Manual of the Illness Attitude Scales. New Mexico: University of New Mexico, Department of Psychiatry, School of Medicine; 1987.

7. Williams JMG, Watts FN, MacLeod C, Mathews A. Cognitive Psychology and Emotional Disorders. Oxford, England: John Wiley \& Sons; 1988.

8. Hadjistavropoulos HD, Frombach IK, Asmundson GJ. Exploratory and confirmatory factor analytic investigations of the Illness Attitudes Scale in a nonclinical sample. Behav Res Ther 1999;37:671-684.

9. Barsky AJ, Geringer E, Wool CA. A cognitive-educational treatment for hypochondriasis. Gen Hosp Psychiatry 1988;10:322-327.

10. Kim MK, Lee KS, Kim B, Choi TK, Lee SH. Impact of mindfulnessbased cognitive therapy on intolerance of uncertainty in patients with panic disorder. Psychiatry Investig 2016;13:196-202.

11. Seol SH, Kwon JS, Kim YY, Kim SN, Shin MS. Internet-based cognitive behavioral therapy for obsessive-compulsive disorder in Korea. Psychiatry Investig 2016;13:373-382.
12. Salkovskis PM, Warwick HM. Morbid preoccupations, health anxiety and reassurance: a cognitive-behavioural approach to hypochondriasis. Behav Res Ther 1986;24:597-602.

13. Hedman E, Axelsson E, Andersson E, Lekander M, Ljotsson B. Exposure-based cognitive-behavioural therapy via the internet and as bibliotherapy for somatic symptom disorder and illness anxiety disorder: randomised controlled trial. Br J Psychiatry 2016;209:407-413.

14. Newby JM, Mahoney AEJ, Mason EC, Smith J, Uppal S, Andrews G. Pilot trial of a therapist-supported internet-delivered cognitive behavioural therapy program for health anxiety. Internet Interv 2016;6:71-79.

15. Lucock MP, Morley S. The health anxiety questionnaire. Br J Health Psychol 1996;1:137-150.

16. Beck AT, Emery G, Greenberg RL. Anxiety Disorders and Phobias: A Cognitive Perspective. New York: Basic Books; 2005.

17. Warwick HM, Salkovskis PM. Hypochondriasis. Behav Res Ther 1990; 28:105-117.

18. Lee IH. Factor Structure of the Illness Attitudes Scale (IAS) in a Korean College Sample. J Health Psychol 2004;9:203-216.

19. Morey LC. The Personality Assessment Inventory Manual. Odessa, FL: Psychological Assessment Resources; 1991.

20. Cohen J. Statistical Power Analysis for the Behavioral Sciences. Hillsdale, NJ: L. Erlbaum Associates; 1988.

21. Kim YH, Kim JH, Oh SW, Lim YR, Hong SH. The Korean Personality Assessment Inventory Manual. Seoul: Hakjisa; 2001.

22. Hu Lt, Bentler PM. Cutoff criteria for fit indexes in covariance structure analysis: Conventional criteria versus new alternatives. Structural equation modeling: a multidisciplinary journal 1999;6:1-55.

23. Mulaik SA, James LR, Van Alstine J, Bennett N, Lind S, Stilwell CD. Evaluation of goodness-of-fit indices for structural equation models. Psychol Bull 1989;105:430-445.

24. Browne MW, Cudeck R. Alternative ways of assessing model fit. Sociol Methods Res 1992;21:230-258

25. Kaiser HF. An index of factorial simplicity. Psychometrika 1974;39:3136.

26. Mulaik SA. Parsimony and Model Evaluation. J Experiment Educ 1998;66:266-273.

27. Bewick V, Cheek L, Ball J. Statistics review 12: survival analysis. Critic Care 2004;8:389. 